\title{
OBSERVATIONS OF HII REGIONS AND PLANETARY NEBULAE: THE INFRARED EMISSION BANDS
}

\author{
JESSE D. BREGMAN \\ NASA Ames Research Center
}

\begin{abstract}
Advances in infrared spectrometers and the theory that PAHs are responsible for the infrared emission bands have led to a wealth of new information in recent years. High quality data have shown many weak emission bands which are diagnostic of the material producing the bands. While correlations of the strengths of the narrow bands indicate that a single material can account for all of the narrow bands, independent spatial behavior of the narrow and broad components show that they have different origins. Predictions of the behavior of the spectra based on laboratory data have been confirmed observationally, strengthening the theory that PAH molecules are the origin of the infrared emission bands.
\end{abstract}

\section{INTRODUCTION}

There has been a flood of observations in the last few years sparked by the development of sensitive IR spectrometers operating from 1-14 $\mu \mathrm{m}$, and by the theory that organic compounds (specifically aromatics) are responsible for the series of emission bands known as the Unidentified Infrared (UIR) bands. So many authors have now claimed identifications for these bands that they have been referred to as the over-identified IR bands by the editors of this volume. In this paper, I will refer to the bands as the infrared emission bands. In the limited space available, I will not be able to cover all the work that has been done, but will concentrate on:

1. The overall spectrum and the separate components.

2. Variations, both spatially and spectrally, and what we have learned from them.

\section{A QUICK HISTORY: WHERE IT ALL STARTED}

Gillett et al. (1973) obtained spectra of NGC 7027 and BD $+30^{\circ} 3639$ to investigate the cause of excess $10 \mu \mathrm{m}$ emission in planetary nebulae which had been observed photometrically. Besides atomic emission lines, they also observed a strangelyshaped continuum and emission features at 8.6 and $11.3 \mu \mathrm{m}$. They suggested that carbonates could account for the $11.3 \mu \mathrm{m}$ band, which Bregman and Rank (1975) confirmed by comparing a higher spectral resolution spectrum of NGC 7027 with laboratory data of carbonates. However, a subsequent observational search for another strong carbonate band at $7 \mu \mathrm{m}$ (Russell et al., 1977) failed to confirm 


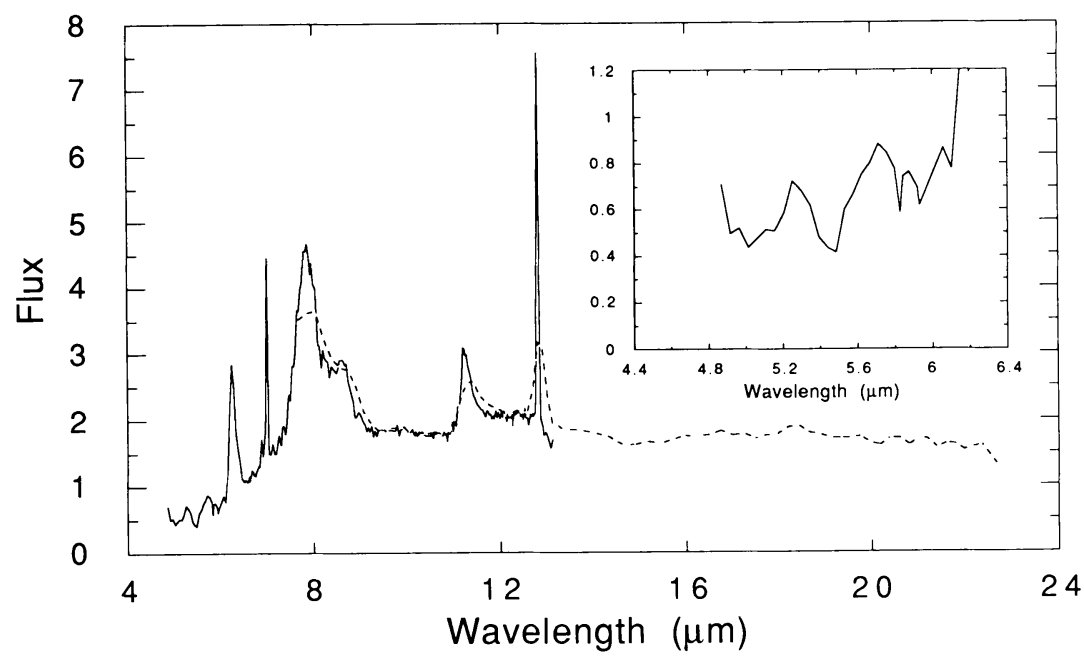

Fig. 1. The 4.6-24 $\mu \mathrm{m}$ spectrum of the planetary nebula $\mathrm{BD}+30^{\circ} 3639$. Data was combined from Witteborn et al. (1989a, 10-13.2 $\mu \mathrm{m}$, solid line), Allamandola et al. (1988, 4.6-10 $\mu \mathrm{m}$, solid line) and the IRAS LRS (broken line). Flux is in units of $10^{-16} \mathrm{~W}-\mathrm{cm}^{-2}-\mu \mathrm{m}^{-1}$. Emission lines from [ArII], $6.98 \mu \mathrm{m}$, and [NeII], $12.78 \mu \mathrm{m}$, are present along with narrow emission features $(5.2,5.6,6.2$, 6.9, 7.7, 8.6, and $11.3 \mu \mathrm{m}$ ). The inset show an expansion of the $5.2 \mu \mathrm{m}$ region. The presence of the $5.2 \mu \mathrm{m}$ band was predicted based on laboratory spectra of PAHs. Note the absence of strong narrow features longward of $11.3 \mu \mathrm{m}$.

the identification. PAH (polycyclic aromatic hydrocarbon) molecules were not yet suggested as the source of the emission bands, and the discrepancy of the position of the "NeII" line in NGC 7027, which appears at $12.7 \mu \mathrm{m}$ and distinctly shortward of the NeII line in $\mathrm{BD}+30^{\circ} 3639$, was not noted. We now would identify it with the $H$-trio band of PAHs.

\section{THE INFRARED SPECTRUM}

\subsection{GENERAL DESCRIPTION}

The spectrum is best described as a combination of broad and narrow components. The broad components are a series of emission features, each a few microns wide, that combine to form the "continuum". Riding on top of the broad features are a series of narrow emission bands which all have the same general asymmetric shape, a sharp rise on the short wavelength side and a wing on the long wavelength side. These features are shown in Figure 1, a spectrum of $\mathrm{BD}+30^{\circ} 3639$ which is a combination of data from Witteborn et al. (1989a, 10-13.2 $\mu \mathrm{m})$, Allamandola et al. $(1988,4.6-10 \mu \mathrm{m})$, and the IRAS LRS data. Note that there are no strong narrow features longward of $13 \mu \mathrm{m}$. There is at least one object, HD44179, that is different from all other objects observed to date, in that it has a fairly symmetrical $11.3 \mu \mathrm{m}$ band (Bregman, 1977; Witteborn et al., 1989a). 
Geballe et al. (1985) show $\mathrm{BD}+30^{\circ} 3639$ from 3-4 $\mu \mathrm{m}$. There is a strong narrow band at $3.3 \mu \mathrm{m}$ with superimposed the $H[9-5]$ emission line, a weaker narrow band at $3.4 \mu \mathrm{m}$, and a broad pedestal extending from at least 3-3.6 $\mu \mathrm{m}$. High spectral resolution data does not show any substructure in the bands, consistent with earlier work by Tokunaga and Young (1980).

Cohen et al. (1985) assembled an average LRS spectrum of non-emission line objects that showed the broad 10-14 $\mu \mathrm{m}$ component very well, and the previously observed but unrecognized band near $12.7 \mu \mathrm{m}$. They suggested that the broad feature could be due to a series of overlapping narrow bands from PAHs with different numbers of neighboring $H$ atoms on the periphery of the molecules. In the PAH model, the $11.3 \mu \mathrm{m}$ band is due to a $C-H$ bend, and the exact wavelength of this mode depends on the number of adjacent $H$ atoms on one ring (cf. Allamandola et al., 1985; Léger and Puget, 1984). Subsequent data obtained across the Orion Bar (Bregman et al., 1988) show that the $11.3 \mu \mathrm{m}$ narrow band has a different spatial distribution than the broad underlying component, making it unlikely that overlapping bands from small PAH molecules could produce the broad feature.

\subsection{CORRELATIONS}

If the same material is responsible for all of the narrow and broad bands, then all the band strengths should vary in unison. For the narrow bands, Cohen et al. (1986, 1988) showed that band-band correlations are very good. In particular, the 7.7 and $6.2 \mu \mathrm{m}$ bands, which have both been assigned to $C-C$ modes in PAHs and thus should correlate very well, do have a very tight correlation. The $3.3,8.6$, and 11.3 $\mu \mathrm{m}$ bands have been assigned to $C-H$ modes, and need not correlate quite as well since they are affected in different ways by the configuration of the molecules. The data show that while the correlations are good, they are not as tight as for the 6.2 vs. $7.7 \mu \mathrm{m}$ bands. Cohen et al. (1986) also found a striking correlation between the energy contained in the $7.7 \mu \mathrm{m}$ band relative to the total energy emitted in the infrared (integrated band energy/total from IRAS) and the $C / O$ ratio, suggesting that the material responsible for the emission bands is carbon-based.

The database assembled by Cohen et al. (1988) has allowed an examination of band ratios versus object type. Figures 2 and 3 show ratios of several bands to the $7.7 \mu \mathrm{m}$ band for three classes of objects, planetary nebula (PN), reflection nebula (RN), and HII regions (HII). Error bars indicate the range for each type. While the trends are not strong, there are some significant differences among the object types. The ratio of $3.3 / 7.7$ is largest in HII regions, while the ratio of $6.2 / 7.7$ is smallest in planetary nebulae. The explanation for these variations is unclear, but one possibility for the lower 6.2/7.7 ratio in planetary nebulae is that this is new material, while the material in HII regions and reflection nebulae was processed in the interstellar medium before being incorporated into these objects. Also, as we will see later, the position of the $7.7 \mu \mathrm{m}$ band is different in planetary nebulae than in HII regions or reflection nebulae. 


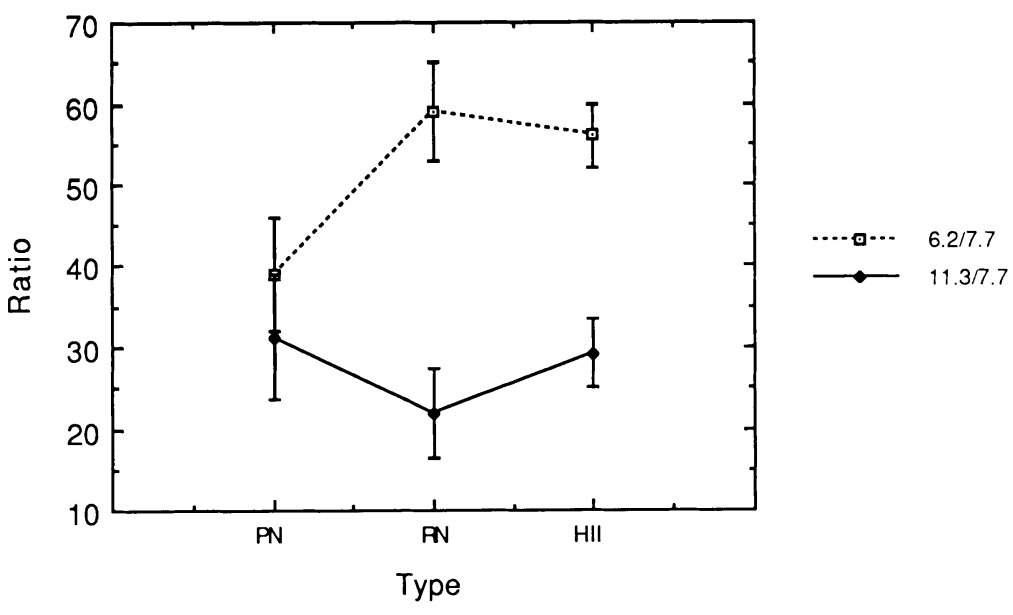

Fig. 2. The ratios of the strong emission bands at 6.2 and $11.3 \mu \mathrm{m}$ bands to the $7.7 \mu \mathrm{m}$ band are shown versus object type. $P N=$ planetary nebula, $R N=$ reflection nebula, and HII=HII region. The error bars indicate the range observed in a class. While the $11.3 \mu \mathrm{m}$ band does not vary among the object types, the $6.2 \mu \mathrm{m}$ band is weaker in planetary nebulae than in reflection nebulae or HII regions. The difference might be explained in terms of the history of the material, since in planetary nebulae the material has just been formed, while in reflection nebulae and HII regions, the material has been incorporated from the interstellar medium and thus has been subjected to an aging process (Cohen et al., 1988).

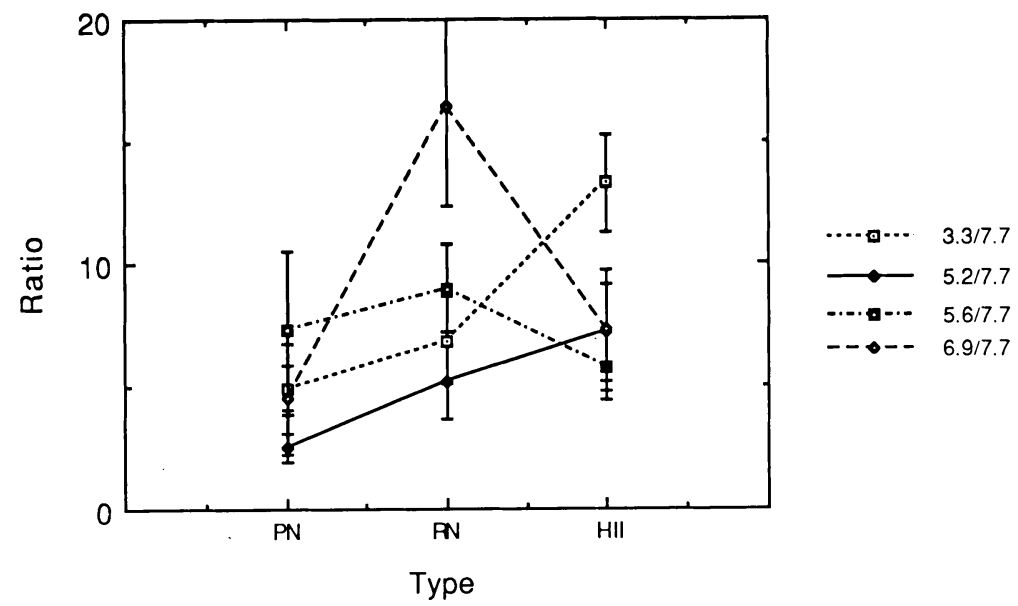

Fig. 8. Ratios for the weak bands to the $7.7 \mu \mathrm{m}$ band are shown versus object type (types same as in Figure 2). A significant deviation is shown for the $3.3 \mu \mathrm{m}$ band, which is stronger in HII regions than in the other two types. The $6.9 \mu \mathrm{m}$ band may be stronger in reflection nebulae than the other types. 


\section{THE OBSERVATIONAL EVIDENCE FOR PAHS OR KNOWING WHAT TO LOOK FOR IN THE DATA}

\subsection{SPECTRAL DATA}

For about ten years, there was no good identification for the material emitting the IR emission bands. Then, Sellgren (1984) observed the features in reflection nebulae along with a $1000 \mathrm{~K}$ continuum. Since the radiation field from the exciting star did not have enough energy to produce the observed temperature, Sellgren suggested a non-thermal mechanism; high temperature spikes of very small ( $\approx 5-10 \AA$ ) grains upon absorption of individual ultraviolet photons. While this model explained the continuum, it did not explain the origin of the narrow emission features. Duley and Williams (1981) noted the coincidence of the $3.3 \mu \mathrm{m}$ emission band with aromatic molecules, and suggested carbon groups attached to small dust grains. Léger and Puget (1984) and Allamandola et al. (1985) carried this line of reasoning to its conclusion and suggested PAHs, large free molecules. This suggestion has implications that can be tested observationally (see the paper by Allamandola, 1989, and references therein, for a full discussion). One of the implications is that if there is a mixture of different PAH molecules contributing to the emission, then the bands

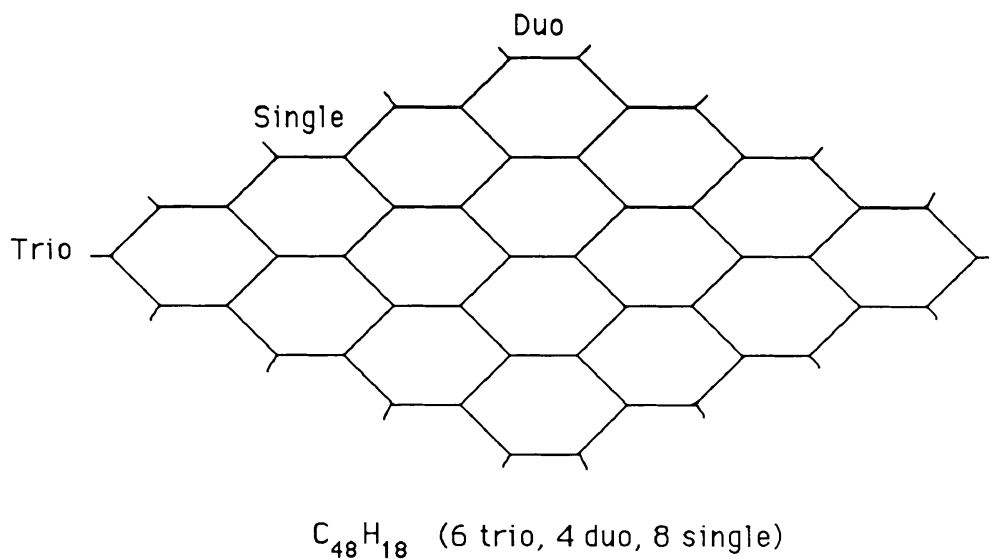

Fig. 4. A schematic representation of a PAH molecule with the single, duo, and trio $H$ atoms on the edge of the molecule indicated.

should not always occur at the same wavelength. In particular, the wavelength of the out of plane $C-H$ bending mode varies from 11-15 $\mu \mathrm{m}$ depending on the number of adjacent $H$ atoms (see Figure 4). The position of the $7.7 \mu \mathrm{m}$ band also varies depending on the size and geometry of the molecule. Also, in many labora- 


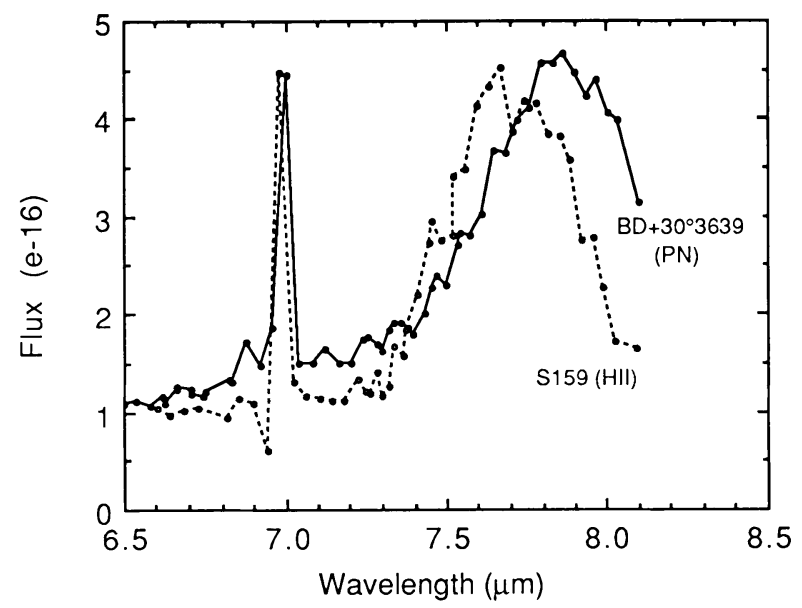

Fig. 5. Spectra of $\mathrm{BD}+30^{\circ} 3639$ (planetary nebula) and S159 (HII region) from 6.5-8.1 $\mu \mathrm{m}$ obtained with the FOGS spectrometer from the Kuiper Airborne Observatory. A strong emission line from [ArII] is present at $6.98 \mu \mathrm{m}$. HII regions and reflection nebulae have a strong component at $7.6 \mu \mathrm{m}$, which is absent or weak in planetary nebulae. HII regions do have the $7.8 \mu \mathrm{m}$ component, but it can be weaker than the $7.6 \mu \mathrm{m}$ component. The flux scale is in units of W-cm ${ }^{-2}-\mu \mathrm{m}^{-1}, \mathrm{and}^{-1}$ errors are typically $5 \times 10^{-18}$.

tory spectra of PAHs, a band is present at $5.2 \mu \mathrm{m}$. Thus, there were at least three predictions based on the association of the emission bands with PAHs that could be tested observationally.

Broad wavelength coverage and high quality data at increasing spectral resolution led to the discovery of many weak bands. In the 3-4 $\mu \mathrm{m}$ region de Muizon et al. (1986) found several weak bands longward of the $3.3 \mu \mathrm{m}$ band. Bregman et al. (1983) had found weak bands at 5.6 and $6.9 \mu \mathrm{m}$ in NGC 7027 that were later found in many different objects (Cohen et al., 1986, 1988). Sandford et al., 1988 (and Witteborn et al., 1989b) reported finding weak bands longward of 11.3 (duo and trio modes) while Roche et al. (1988) found that the trio band in the Orion Bar correlated with the $11.3 \mu \mathrm{m}$ band. Allamandola et al. (1988) observed the 5.2 $\mu \mathrm{m}$ band in $\mathrm{BD}+30^{\circ} 3639$ that had been predicted based on laboratory spectra of PAHs.

Bregman et al. (1987) showed that the $7.7 \mu \mathrm{m}$ band has at least two components, one at $7.6 \mu \mathrm{m}$ and a second at $\approx 7.8 \mu \mathrm{m}$. Figure 5 presents more recent high resolution data that shows this quite clearly. The $7.6 \mu \mathrm{m}$ component is strongest in HII regions and reflection nebulae, while some planetary nebulae (i.e. $\mathrm{BD}+30^{\circ} 3639$ ) show no evidence for a 7.6 $\mu \mathrm{m}$ component. The $7.6 \mu \mathrm{m}$ band shape can be determined by ratioing an HII region to a planetary nebula. Figure 6 shows this ratio for two different cases. Some objects (i.e. NGC 7027) show a weaker $7.6 \mu \mathrm{m}$ component than observed in HII regions, but stronger than in planetaries like $\mathrm{BD}+30^{\circ} 3639$.

Figure 5 shows the large differences of the $7.7 \mu \mathrm{m}$ band observed between the two classes of objects. It is even possible to classify sources based on the position of this band. In their low spectral resolution data $(\lambda / \Delta \lambda \approx 50)$, Cohen et al. (1988) find that the central wavelength varies among different types of objects, with the mean central wavelength and standard deviation as a function of object type as 


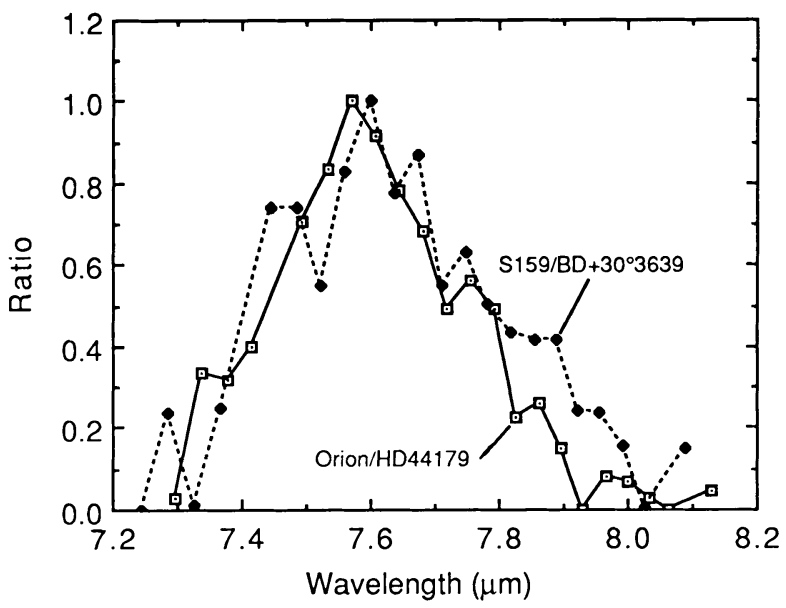

Fig. 6. The $7.6 \mu \mathrm{m}$ component has been separated from the broader feature by ratioing an HII region to a planetary or protoplanetary nebula and then removing the residual slope between 7.3 and $8.0 \mu \mathrm{m}$. This procedure assumes that there is no extra emission shortward of $7.3 \mu \mathrm{m}$ or longward of $8.0 \mu \mathrm{m}$.

follows: $\mathrm{PN}$ 's $=7.78 \pm 0.03 \mu \mathrm{m}, \mathrm{RN}$ 's $=7.67 \pm 0.02 \mu \mathrm{m}$, and $\mathrm{HII}=7.64 \pm 0.02 \mu \mathrm{m}$.

\subsection{SPATIAL DATA}

Maps of regions with emission bands have provided clues to the kinds of environments in which the material responsible for the bands can survive, and how well the features correlate spatially with each other. Maps through narrow bandpass filters were obtained by Bentley (1982) and Bentley et al. (1984) of NGC 7027 and $\mathrm{BD}+30^{\circ} 3639$. They clearly showed that the distribution of the narrow features was different than the continuum, with the continuum centrally condensed while the features peaked towards the edge of the nebula. Aitken and Roche (1983) confirmed this result with a series of spectra obtained in a cut across NGC 7027. Goebel et al. (1987) and Tresh-Fienberg (1985) obtained images of NGC 7027 using filters in front of two dimensional arrays. Their filter bandwidths were narrower than those used by Bentley, and showed that the spatial distribution varied with wavelength.

Geballe et al. (1988) and Tokunaga et al. (1988) have shown that the ratio of the 3.3/3.4 $\mu \mathrm{m}$ bands varies spatially in both HD44179 and the Orion Bar. The central part of HD44179, where there is a harsh UV environment, shows a narrow $3.3 \mu \mathrm{m}$ and no $3.4 \mu \mathrm{m}$ band, while 5 arcseconds away from the star, the $3.3 \mu \mathrm{m}$ band is the same width as observed in other sources and a $3.4 \mu \mathrm{m}$ band is present.

The Orion Bar is a well studied, nearly edge-on HII region-molecular cloud interface. Its spectrum is typical of emission band objects, showing narrow and broad features. Bregman et al. (1988) obtained spectra from 3-13 $\mu \mathrm{m}$ at several positions across the Bar. They observe emission lines from hydrogen (Brackett Alpha) and NeII $(12.78 \mu \mathrm{m})$, which define the edge of the interface, and both the narrow and broad emission bands. The emission bands originate from a thick $\left(\approx 15^{\prime \prime}\right.$ or $10^{17}$ 


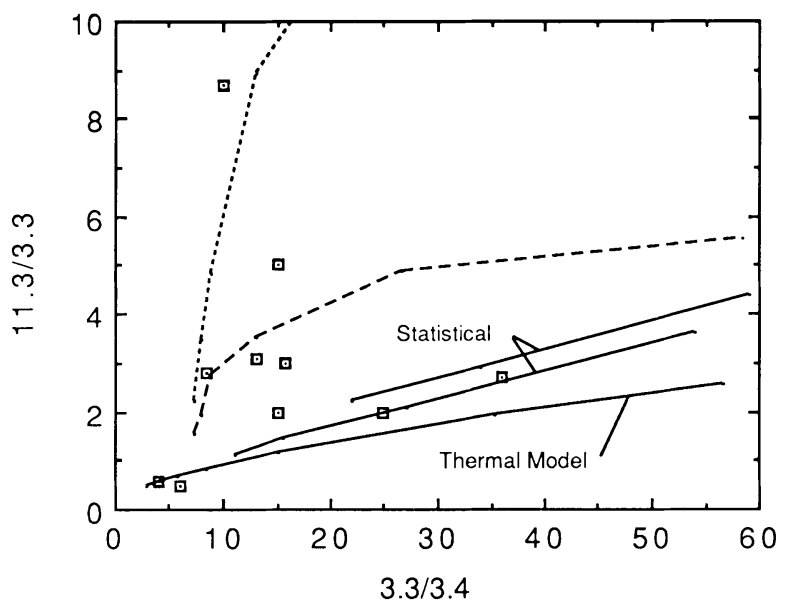

Fig. 7. The ratio of $11.3 / 3.3$ versus $3.3 / 3.4$ is a powerful method for comparing data (points) with theory (lines) since assumptions about the energy of absorbed UV photons or number of $C$ atoms in the molecules is removed. The solid lines show the ratios expected for a single size of molecule, while the broken lines show the ratios expected for a bimodal size distribution of molecules.

$\mathrm{cm}$ ) region just outside the HII region, while the broad bands are more uniformly distributed. The narrow and broad features have different distributions consistent with the narrow features originating from molecules which can be destroyed by UV, and the broad features arising from larger particles. Sellgren (1981) had observed that the $3.3 \mu \mathrm{m}$ band also peaked on the edge of the HII region. The pedestal under the $3.3 \mu \mathrm{m}$ band has a distribution similar to the $3.3 \mu \mathrm{m}$ narrow feature, not the longer wavelength broad features, implying that the 3-4 $\mu$ m pedestal has the same origin as the narrow bands, different than other broad features. Geballe et al. (1988) showed that the 3.3/3.4 ratio decreases going into the molecular cloud, while Roche et al. (1988) have shown that a $12.7 \mu \mathrm{m}$ band (trio $H$ ) correlates well with the single $H$ band at $11.3 \mu \mathrm{m}$ going across the Bar. These results indicate that the variations in the $3 \mu \mathrm{m}$ region are of a different origin than those in the 11 $\mu \mathrm{m}$ region.

\section{APPLYING THEORY TO OBSERVATIONS}

Allamandola et al. (1985) and Léger and d'Hendecourt (1987) have calculated the sizes of PAH molecules that would give the observed 11.3/3.3 ratios, and Barker et al. (1987) also calculated the sizes based on the 3.3/3.4 ratios and the assumption that the $3.4 \mu \mathrm{m}$ band arose from an excited state. In all cases, the calculations assumed an average excitation energy for the molecules. If the $11.3 / 3.3$ ratio is combined with the 3.3/3.4 ratio, then the resulting plot is independent of assumed molecule size or excitation energy, and can be directly compared with data. Figure 7 shows both data (points) and model calculations. Reasonable agreement between the observations and models (solid lines) is obtained for some data points, while other data points are clearly above the model predictions. Some of the difficulty arises because the $3.3 \mu \mathrm{m}$ data is not obtained with the same size aperture as the 
11.3 $\mu \mathrm{m}$ data. Since the emission is often extended outside the small apertures used at $3 \mu \mathrm{m}$, it is difficult to compare with the longer wavelength results. However, some objects which lie above the theoretical curves do not suffer from this problem. Their ratios may be explained if the emission bands arise from a mixture of molecular sizes. The smallest molecules will contribute mainly to the 3.3 and $3.4 \mu \mathrm{m}$ bands while the largest molecules contribute mainly to the $11.3 \mu \mathrm{m}$ band. The theoretical curves obtained by using bimodal size distributions are shown as broken lines in Figure 7 . Note that reflection nebulae, which should have the most benign environment, have the smallest $3.3 / 3.4$ ratios and thus appear to have the smallest molecules.

\section{SUMMARY}

Improvements in equipment and observations to test the theory that PAHs are the origin of the infrared emission bands has led to a wealth of new information in the last few years about the emission bands. Predictions based on laboratory data of PAH molecules that a $5.2 \mu \mathrm{m}$ band should be present, that the $7.7 \mu \mathrm{m}$ band should have multiple components, and that additional bands should be present longward of the $11.3 \mu \mathrm{m}$ band have all been confirmed observationally. Making predictions based on a theory and then confirming the predictions is the essence of the scientific method, and is a strong indication that PAHs are indeed the source of the infrared emission bands.

ACKNOWLEDGEMENTS. I wish to thank Martin Cohen, Fred Witteborn, and Lou Allamandola for providing preprints of results, and to Amara Graps for her help in analyzing data.

\section{REFERENCES}

Aitken, D. K., and Roche, P. F. 1983, M. N. R. A. S., 202, 1233.

Allamandola, L. J., Tielens, A. G. G. M., and Barker, J. R. 1985, Ap. J. (Letters), 290, L25.

Allamandola, L. J., Bregman, J. D., Sandford, S. A., Witteborn, F. C., Wooden, D. H., and Rank, D. M. 1988, preprint.

Allamandola, L. J. 1989, in IAU Symposium 195, Interstellar Dust, eds. L. J. Allamandola and A. G. G. M. Tielens, (Dordrecht: Kluwer), p. 129.

Barker, J., Allamandola, L. J., and Tielens, A. G. G. M. 1987, Ap. J., 315, L61.

Bentley, A. 1982, A. J., 87, 1810.

Bentley, A., Hackwell, J. A., Grasdalen, G. L., and Gehrz, R. D. 1984, Ap. J., $278,665$.

Bregman, J. D. 1977, Pub. A. S. P., 89, 335.

Bregman, J. D., and Rank, D. M. 1975, Ap. J. (Letters), 195, L125.

Bregman, J. D., Dinerstein, H. L., Goebel, J. H., Lester, D. F., Witteborn, F. C., and Rank, D. M. 1983, Ap. J., 274, 666.

Bregman, J. D., Allamandola, L. J. , Tielens, A. G. G. M., Witteborn, F. C., Rank, D. M., and Wooden, D. H. 1987, in Summer School on Interstellar Processes, Abstracts of Contributed Papers, eds. Hollenbach D. and Thronson, H., (NASA TM 88342), p. 117.

Bregman, J. D., Allamandola, L. J ., Tielens, A. G. G. M., Geballe, T., and Witteborn, F. C. 1988, Ap. J., submitted.

Cohen, M., Tielens, A. G. G. M., and Allamandola, L. J. 1985, Ap. J. (Letters), 299, L93.

Cohen, M., Allamandola, L. J., Tielens, A. G. G. M., Bregman, J. D., Simpson, J. P., Witteborn, F. C., Wooden, D. H., and Rank, D. 1986, Ap. J., 302, 737.

Cohen, M., Tielens, A. G. G. M., Bregman, J. D., Witteborn, F. C., Rank, D. M., Allamandola, L. J., Wooden, D. H., and de Muizon, M. 1988, Ap. J., submitted. 
de Muizon, M., Geballe, T. R., d'Hendecourt, L. B., and Baas, F. 1986, Ap. J. (Letters), s06, L105.

Duley, W. W., and Williams, D. A. 1981, M. N. R. A. S., 196, 269.

Geballe, T. R., Lacy, J. H., Persson, S. E., McGregor, P. J., and Soifer, B. T. 1985, Ap. J., 292, 500.

Geballe, T. R., Tielens, A. G. G. M., Allamandola, L. J., Morehouse, A., and Brand, P. W. J. L. $1988, A p$. J., in press.

Gillett, F. C., Forrest, W. J., and Merrill, K. M. 1973, Ap. J., 183, 87.

Goebel, J. H., Rank, D. M., and Cohen, M. 1987, in Infrared Astronomy with Arrays, eds. WynnWilliams, C. G., and Becklin, E. E., (Honolulu: University of Hawaii), p. 305.

Léger, A. and d'Hendecourt, L. B. 1987, in Polycyclic Aromatic Hydrocarbons and Astrophysics, eds. Léger, A., d'Hendecourt, L. B., and Boccara, N., (Dordrecht: Reidel), p. 223.

Léger, A., and Puget, J. L. 1984, Astr. Ap., 197, L5.

Roche, P., Aitken, D. A., and Smith, C. H. 1988, preprint.

Russell, R. W., Soifer, B. T., and Willner, S. P. 1977, Ap. J. (Letters), 217, L149.

Sandford, S. A., Witteborn, F. C., Bregman, J. D., Cohen, M., and Allamandola, L. J. 1988, $B A A S, 19,952$.

Sellgren, K. 1981, $A p$. J., 245, 138.

. 1984, Ap. J., 277, 623.

Tokunaga, A. T., and Young, E. T. 1980, Ap. J. (Letters), 237, L149.

Tokunaga, A. T., Nagata, T., Sellgren, K., Smith, R. G., Onaka, T., Nakada, Y., Sakata, A., and Wada, S. 1988, Ap. J., 328, 709 .

Tresch-Fienberg, R. 1985, Ph. D. thesis, Harvard.

Witteborn, F. C., Sandford, S. A., Bregman, J. D., Allamandola, L. J., Wooden, D. H., Cohen, M., Graps, A. L. $1989 a, A p$. J. in press.

Witteborn et al. $1989 b$, in Interstellar Dust Contributed Papers, eds. A. G. G. M. Tielens and L. J. Allamandola, NASA CP-3036. 